\title{
Evolution of Gold Nanostructures on Graphene
}

\author{
Recep Zan, Ursel Bangert, * Quentin Ramasse, and Konstantin S. Novoselov
}

Ever since the first experimental observation of graphene, ${ }^{[1]}$ much of graphene research has concerned the control of its intrinsic properties. Attempts to achieve this have included doping or functionalizing either with metals, ${ }^{[2,3]}$ molecules, ${ }^{[4]}$ or exposure to a hydrogen plasma. ${ }^{[5]}$ Metal-graphene interactions have been much studied theoretically, in terms of the specific sites of the metals on the benzene ring, their binding energies etc. So, for example, center-ring positions ( $\mathrm{H}$ sites) are predicted as preferred locations for most metals (e.g., Ti, $\mathrm{Fe}$ ), corner sites directly above $\mathrm{C}$ atoms ( $\mathrm{T}$ sites) for $\mathrm{Sb}, \mathrm{Sn}$, and $\mathrm{Ni}$, and bridge sites above $\mathrm{C}-\mathrm{C}$ bonds (B sites) for $\mathrm{Pd}$, $\mathrm{Cr}$, and Pt. ${ }^{6-10]}$ Some predictions are even in contradiction with each other because of different approximations for the calculations; the local density and the generalized gradient approximations lead to different binding energies and thereby to different sites. For $\mathrm{Au}$ atoms, for example, $\mathrm{T}$ sites are predicted by the former and B sites by the latter method. ${ }^{[11]}$ Furthermore, arbitrary variables used in the calculation, such as cutoff energies ${ }^{[12]}$ and size of the supercell, ${ }^{[13]}$ can affect the result of density functional theory (DFT) calculations. Also, some calculations predict gold to dope graphene in an n-type manner, whereas others predict p-type doping effects. ${ }^{[11,14,15]}$ The contradictions in the calculations have so far not been resolved by experimental studies. For example, McCreary et al. ${ }^{[16]}$ predict $n$-type doping of graphene for gold deposition, whereas p-type doping is predicted from Raman spectroscopy studies of the gold-graphene interaction. ${ }^{[17]}$ The goldgold interaction is expected to be significantly stronger than the gold-graphene interaction (for this reason gold is highly mobile on graphene). ${ }^{[12]}$ This was experimentally confirmed by Gan et al., ${ }^{[14]}$ who observed gold clusters (not individual atoms) on graphene. Indeed, on the grounds of DFT calculations Pinto et al. ${ }^{[15]}$ propose that $\mathrm{Au}$ does not dope singlelayer graphene at all, but bilayer graphene. However, as theoretically predicted and experimentally confirmed, point

\author{
R. Zan, Prof. K. S. Novoselov \\ School of Physics and Astronomy \\ The University of Manchester \\ Manchester M13 9PL, UK \\ Dr. U. Bangert \\ School of Materials \\ The University of Manchester \\ Manchester M13 9PL, UK \\ E-mail: ursel.bangert@manchester.ac.uk \\ Dr. Q. Ramasse \\ SuperSTEM Laboratory \\ STFC Daresbury \\ Keckwick Lane, Daresbury WA4 4AD, UK
}

DOI: 10.1002/smll.201101169 defects in graphene can provide nucleation sites and even mediate substitutional incorporation of metals. ${ }^{[14,18-22]}$

$\mathrm{Au}$ is one of the most widely used metals for a range of graphene applications, including making contacts to circuits, ${ }^{[1]}$ electrochemical catalysis, ${ }^{[23]}$ biosensors, ${ }^{[24]}$ and studying interfaces. ${ }^{[25]}$ So direct observation of gold behavior on graphene-for example, nucleation and coverage-in particular by transmission electron microscopy (TEM), is one of the key requirements to understand the nature of the interaction, and to gain a better understanding of the properties of metal contacts on suspended single-layer graphene and their effect on macroscopic electrical transport. The graphene surface, due to its $\pi$-electron system, is inherently reactive, especially with regard to adsorbing hydrocarbon chains. These in turn are strong competitors as metal nucleation sites and will impose impedances on electrical transport into the metal, greatly defying the objective of exploiting the outstanding properties, such as the phenomenal carrier mobilities in graphene. On the other hand, "extrinsic" nucleation sites might prove crucial because of the weak interaction of graphene with metals, as also predicted above. In this case it is essential to identify the right amount and nature of essential extrinsic nucleation sites, for example, hydrocarbon contamination, in order to prevent deterioration of the electrical properties. Several groups have studied the deposition of gold nanoparticles on functionalized graphene in solution. ${ }^{[26-28]}$ These studies point out that oxy-functional groups, which act as nucleation sites, facilitate seeding and growth of Au clusters. Herein, we explore the structural evolution of gold films evaporated on graphene, which was obtained via exfoliation or chemical vapor deposition (CVD) growth methods.

Figure 1 shows the results of various gold depositions: we never observed gold atoms on single or decoupled (turbostratic) layers; however, gold clusters and nanocrystals form in the hydrocarbon contamination, revealed by the wormlike contrast in the high-resolution TEM images. This demonstrates the extreme mobility of gold on pure graphene, similar to observations by Charlier et al. of Au-doped carbon nanotubes. ${ }^{[19]}$

The gold cluster density increases initially with increasing evaporated amount of $\mathrm{Au}$, and at a nominal gold thickness of $>1 \mathrm{~nm}$ clusters start to coalesce as a result of continued evaporation. The images in Figure $1 \mathrm{a}-\mathrm{d}$ demonstrate this in samples evaporated with $<0.1,0.12$, and $2.12 \mathrm{~nm}$ of gold, respectively.

Figure 2 shows merging of clusters. The clusters stay surrounded by contamination, which indicates that even at high nucleation densities preferred nucleation points remain within the contamination. There are, however, lighter areas within the clusters. This is due to the fact that the clusters 


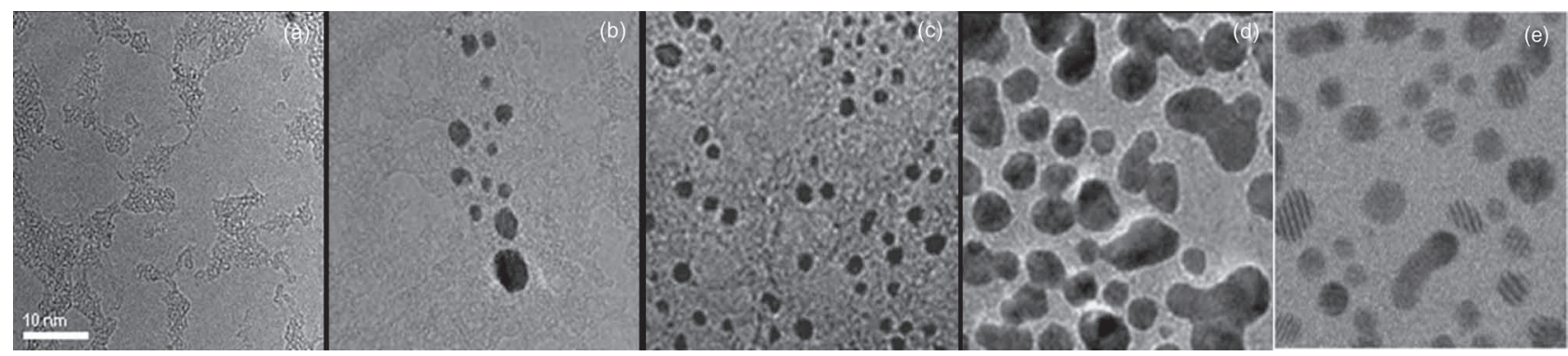

Figure 1. a-d) TEM images of different gold coverages of the same graphene sample, which was repeatedly exposed to gold evaporation amounting to nominal thicknesses of $<0.1,0.12$, and $2.12 \mathrm{~nm}$. a) Sparse coverage and b) the occasional group of clusters at $<0.1 \mathrm{~nm}$ gold thickness. c) Higher cluster densities at $0.12 \mathrm{~nm}\left(1 \mathrm{~s}\right.$ evaporation with $1.2 \AA \mathrm{s}^{-1}$ evaporation rate) and d) coalescence of clusters at $2.12 \mathrm{~nm}(2 \mathrm{~nm}$ evaporation on the same sample) gold thickness. e) Scanning TEM (STEM) bright-field (BF) image showing coverage following a single gold evaporation to nominally $0.5 \mathrm{~nm}$ thickness. Scale bar: $10 \mathrm{~nm}$ in all images.

overlay clean graphene patches. Two such positions are encircled in white in Figure 2a; the left one occurs at the coalescence front of two particles, the right-hand one in the middle of a particle. The gold layer is also thinner in these regions, thus suggesting that gold atoms prefer deposition on existing gold clusters before they are finally forced to spread across, or indeed bridge, clean graphene areas. Lattice images are less clear in these "bridging" regions, which suggests disturbance of the electron wave function possibly due to decoupled, randomly oriented lattices between the two materials. Particles contain planar faults in the coalescence planes or undergo twinning (top and bottom arrows in Figure 2b).

Single gold atoms can only be observed on few-layer graphene films, where the bonding contribution from subsurface layers appears to prohibit their diffusion and increases interaction with the graphene surface. Figure 3a is a BF (i.e., phase-contrast) STEM image with the beam focused onto the graphene and not onto the gold cluster (which is positioned at the exit surface); hence the gold-atom aggregate is invisible. However, the benzene rings exhibit strong contrast and relatively little noise, and so the BF image can be used as a guide to identify positions in the corresponding, simultaneously acquired high-angle annular dark-field (HAADF) image (Figure 3b), which has inherently much lower intensity, but in which the gold atoms are clearly visible. The loose aggregate (on the right in Figure 3b) with single atoms in its vicinity (some of which have been numbered) has formed as a result of repeated electron-beam scans, during which atoms were dissociated from a larger gold nanocrystal (bright area on the left in Figure 3b). To correlate the BF and HAADF contrast with the location of the benzene rings, STEM image simulations were carried out (three-layer simulations at $-5 \mathrm{~nm}$ defocus giving the best match) and are shown as insets in Figure $3 a$ and $b$. The benzene ring centers of the top layer correspond to bright patches in the BF image (a) and to dark patches in the HAADF image (b). The model structure (black benzene rings) is overlaid in identical locations on both images. Hence $\mathrm{C}$ atoms constitute dark contrast in $\mathrm{BF}$ and bright contrast in HAADF images. By comparing locations of gold atoms in the HAADF image with identical positions in the BF image (circles), the sites can be identified as T sites.

As mentioned before, these single gold atoms are always found exclusively in the close vicinity of very thin gold clusters, from which they have presumably been separated by the scanning electron beam. On few-layer graphene, small gold clusters were found to sit directly on the pristine surface, thereby giving rise to moiré effects. The wide fringes in the cluster in Figure 4a arise from rotational moiré effects, where the moiré fringe spacing depends on the difference in lattice spacing between gold and graphene as well as on the rotation angle of the two lattices with respect to each other. The largest proportion of gold clusters shows moiré fringe spacing as seen in Figure 4a. Analysis of the patterns shows

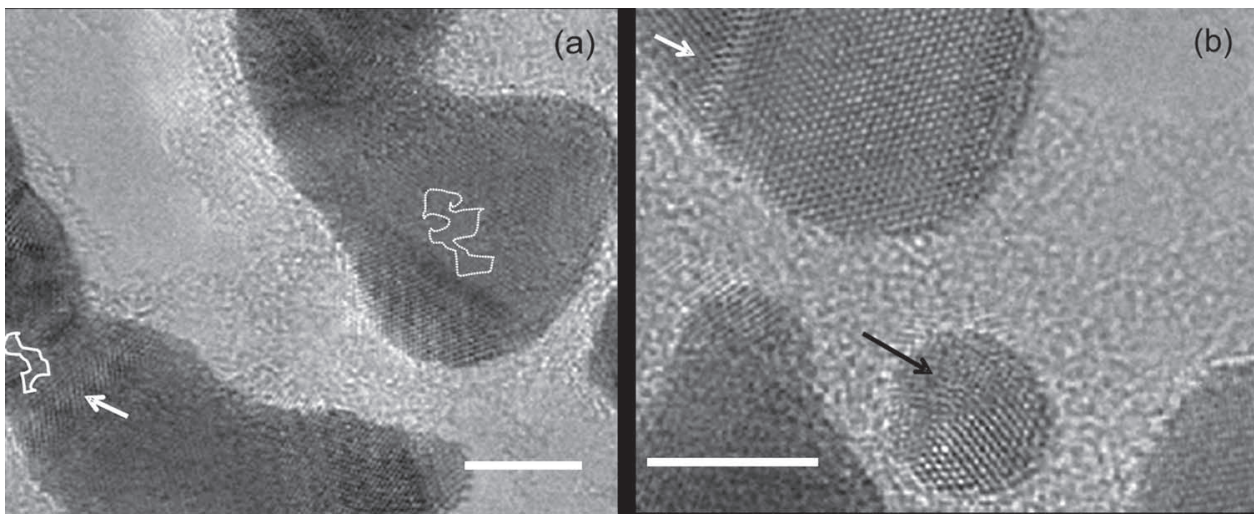

Figure 2. Coalesced clusters after evaporation of $2.12 \mathrm{~nm}$ gold showing a) variations in thickness and relative crystallographic orientations and b) planar faults such as stacking faults (white arrows) and twin boundaries (black arrow). Scale bar: $5 \mathrm{~nm}$ in both images. 

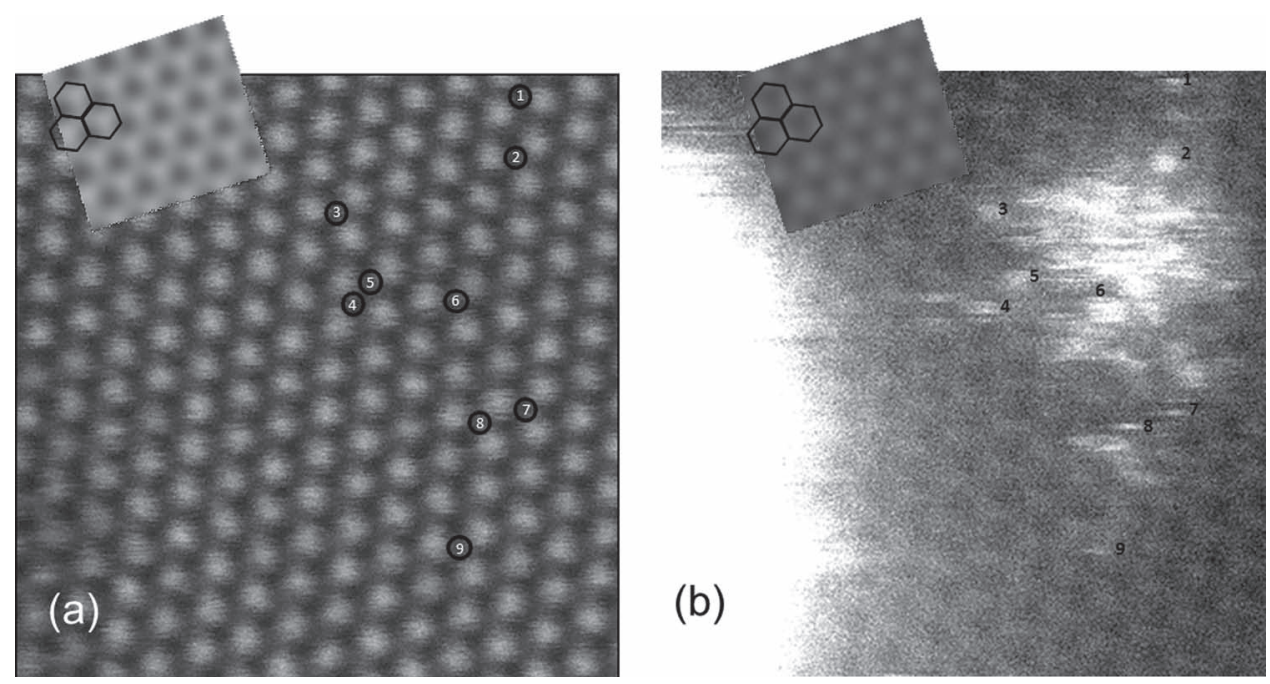

Figure 3. a) BF and b) HAADF STEM images of $0.5 \mathrm{~nm}$ gold evaporated on few-layer graphene. In the HAADF image individual gold atoms can be seen to have separated from a gold cluster on the left-hand side. Identical positions of the benzene rings are marked in black in the image simulations overlaid on the experimental images, which show that bright contrast in the BF image and dark contrast in the HAADF image correspond to the centers of benzene rings. Single-atom positions 1-9 are marked with numbers just to the right of the bright atoms in the HAADF image; identical places are marked by circles with the numbers inside in the BF image, which shows that Au atoms sit on T sites on the sample surface. All images represent raw, unfiltered data. The frame width is $3 \mathrm{~nm}$.

that the graphene $\{100\}$-type planes with spacing $2.1 \AA$ conform to the $\{200\}$ planes of the gold crystals $(2.035 \AA)$. The latter present themselves in 110 orientation on the graphene surface and, due to the mismatch, undergo rotations $\left(\approx 5^{\circ}\right)$. Figure $4 \mathrm{~b}$ is a noise-filtered HAADF image, obtained using the circular low-pass filter in the fast Fourier transform (FFT) in the inset. The image in Figure $4 \mathrm{~b}$ is taken simultaneously with the BF image in Figure 4a. Both the graphene and the gold lattice can be seen in the HAADF image. Sections of the two lattices are shown enlarged next to each other in the
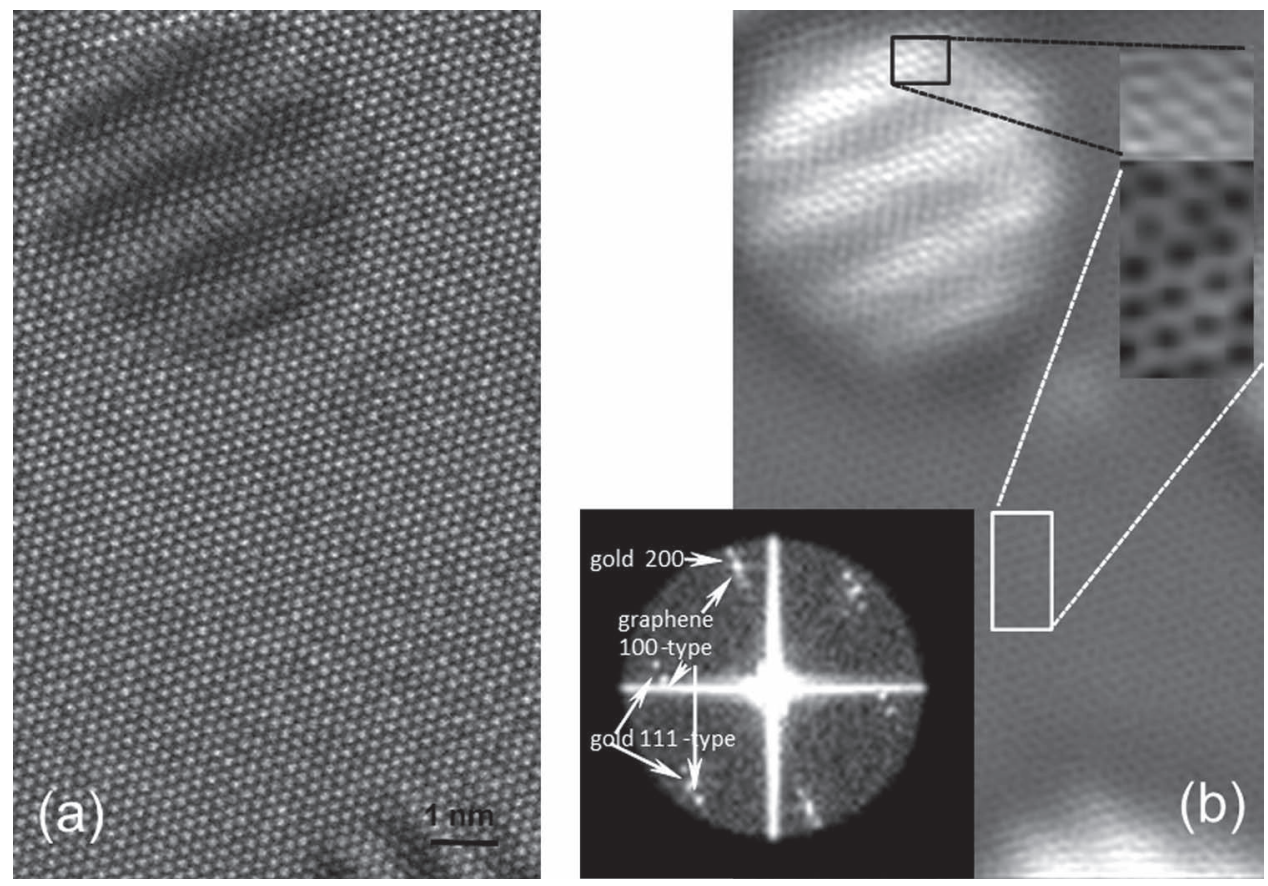

Figure 4. a) Atomic-resolution BF STEM image of few-layer graphene with gold clusters revealed by moiré effects. b) Noise-filtered HAADF STEM image of the same area as in (a), which shows both the graphene and the gold lattice. The bottom inset is the FFT of the raw HAADF image showing the low-pass filter that was used to obtain the image in (b). The FFT also reveals rotation of the gold $\{111\}$ planes and orientation coincidence of the gold 200 planes and the graphene planes. The top insets show the enlarged gold and graphene lattice structures, and reveal directly the gold and graphene lattice orientation relationship. 


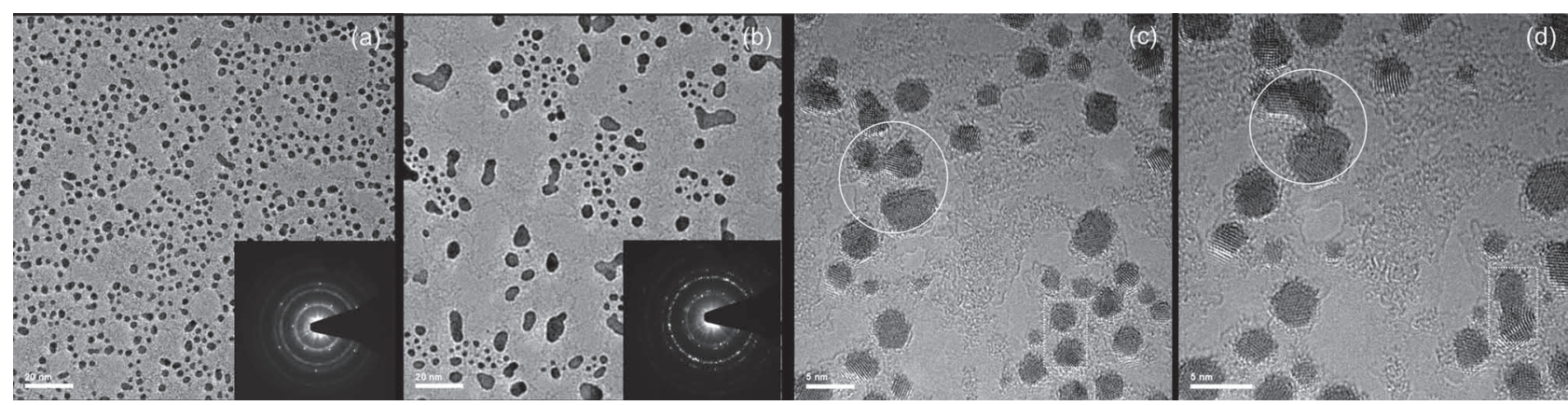

Figure 5. a, b) TEM images of $0.2 \mathrm{~nm}$ gold evaporated onto hydrogenated and pristine graphene shown at the same magnification (scale bar: $20 \mathrm{~nm}$ ). The corresponding diffraction patterns are shown as insets. c, d) Images of gold evaporated onto hydrogenated graphene, taken in subsequent scans, and showing how gold clusters have coalesced (the solid circles and dashed rectangles; scale bar: $5 \mathrm{~nm}$ ).

inset: the gold $\{111\}$ planes are slightly rotated with respect to two sets of the graphene $\{100\}$ planes; this achieves maximum coincidence of plane spacings in the gold [110] and the graphene [001] zones. Further matching is achieved by the gold 200 planes being oriented parallel to the third set of the graphene $\{100\}$ planes, as can be observed in the FFT inset in Figure $4 \mathrm{~b}$; the spacings of these planes are the most similar in the two structures. Rotation will, however, reduce contact points between gold and graphene.

Further to doping with metals, an alternative way to modify and functionalize graphene is by dosing, for example with hydrogen and fluorine. ${ }^{[5,29,30]}$ Hydrogenation breaks graphene $\mathrm{sp}^{2}$ bonds and leads to $\mathrm{sp}^{3}$ bond formation, thereby opening up a band gap. We carried out gold deposition on graphene surfaces that had been hydrogenated. The reason for this experiment was to investigate whether hydrogenated surfaces might increase the sticking probability for gold atoms. To conduct a fair comparison, gold evaporation was performed with the same amount $(0.2 \mathrm{~nm})$ and simultaneously on hydrogenated and pristine graphene surfaces. As can be seen in Figure 5a, the hydrogenated sample has a higher gold coverage, and gold cluster distributions and cluster sizes are of higher uniformity than in the pristine graphene sample, as shown in the image in Figure $5 \mathrm{~b}$ taken at the same magnification. In the latter case less than half of the area is covered with gold and cluster sizes vary. The diffraction pattern of the sample in Figure 5a (inset) shows a ring, which arises from small, randomly oriented clusters. In contrast, the diffraction pattern of the pristine sample in Figure 5b (inset) shows distinct spots, which arise from few clusters with bigger sizes. Both diffraction patterns clearly show graphene spots. However, similar to pristine graphene, gold is only retained in the contamination in the hydrogenated sample, as can be seen in the higher-magnification images in Figure $5 \mathrm{c}$ and d.

The above observations can be explained as follows. Gold does not stick to (uncontaminated) graphene surfaces, pristine or hydrogenated. A possible reason why hydrogenmodified graphene does not show an increase in the sticking probability might be due to the fact that hydrogenation takes place predominantly in the contamination; here it appears to provide increased bonding, as it causes the gold to adhere more effectively, hence the higher nucleation rate of gold clusters in the contamination. This might also explain the observable coalescence of gold clusters under the electron beam in the hydrogenated sample, which is not seen in the pristine sample. An example of this process in the hydrogenated sample is shown in Figure $5 \mathrm{c}$ and d; the time elapsed between the acquisition of the images is less than $10 \mathrm{~s}$. Hence, agglomeration of clusters (indicated with solid circles and dashed rectangles in Figure 5c and d) occurs relatively rapidly. In contrast, in the pristine sample coalescence of small clusters has most likely already happened during evaporation; here the process carries on between already coalesced larger clusters after prolonged electron-beam exposure (several minutes), which comprises the unfavorable route of bridging clean graphene patches (see Figure 2a, white shapes). This confirms the aforementioned suggestion that hydrogenation of the contaminants, which appear to act as the "substrate", enables stronger interaction with the gold clusters. Hence a diffusion barrier has to be overcome in the first place-with the aid of the electron beam - to initiate coalescence of gold clusters.

In conclusion, it appears that gold atoms do not adhere to clean single-layer graphene, but at the lowest deposition thickness of $<0.1 \mathrm{~nm}$ form clusters $(2-3 \mathrm{~nm}$ in diameter), which nucleate exclusively in the abundant hydrocarbon surface contamination. Higher deposition thicknesses result in a higher density of clusters. At a thickness of $>1 \mathrm{~nm}$, the density remains rather constant; however, gold particles grow to coalesce. During this stage clean graphene areas inevitably have to be bridged. However, whether and how gold actually bonds with graphene, and whether charge transfer into electrical contacts deposited in this way proceeds, in the largest part, across a macromolecular (hydrocarbon) layer, requires further investigation. Gold thicknesses on the hydrocarbon contamination are much larger, and 3D crystals grow under extended fault and twin formation. 2D gold nanoclusters as well as single gold atoms do, however, adhere to clean surface areas in few-layer graphene. The $2 \mathrm{D}$ clusters here undergo rotation to achieve matching of planes in the gold $\langle 110\rangle$ and graphitic [001] zones, and the sites of single gold atoms were identified as $\mathrm{T}$ sites, that is, on top of carbon atoms. Gold nucleation on hydrogenated graphene surfaces is confined to the contamination; however, the gold coverage is higher than on pristine graphene for the same evaporated amount of gold, with a greater uniformity of cluster size and distribution. This 
is thought to be due to provision of stronger bonding within the hydrogen-modified surface contamination.

\section{Experimental Section}

Two different techniques were employed for graphene preparation: 1) exfoliation from highly oriented pyrolytic graphite (few-layer sample) ${ }^{[1]}$ and 2) the CVD method, in which graphene is grown on copper (monolayer samples). ${ }^{[31]}$ We evaporated various amounts of gold, from less than 0.1 up to $2.12 \mathrm{~nm}$, by using electron-beam and thermal evaporators. Evaporation was performed at room temperature and $\approx 10^{-8}$ mbar pressure. Hydrogenation was carried out by exposing graphene on a TEM grid to a cold hydrogen plasma. For this purpose a low-pressure (0.1 mbar) hydrogen-argon mixture $\left(10 \% \mathrm{H}_{2}\right)$ was used. Because the graphene was freely suspended on the TEM grid, hydrogenation occurred on both surfaces. Graphene samples were kept in the hydrogen plasma for $1 \mathrm{~h}$; the system pressure before plasma formation was about $5 \times 10^{-6} \mathrm{mbar}$. Details about the hydrogenation can be found in the literature. ${ }^{[5]}$

For high-resolution imaging, including atomic-resolution lattice imaging of gold crystals and atoms on the graphene surface, transmission electron microscopy was carried out in stationary mode (TEM) as well as in scanning mode (STEM), the former in a Tecnai F30, the latter in an aberration-corrected dedicated STEM instrument (Daresbury SuperSTEM). Advantage was taken of the HAADF imaging mode, the contrast of which has an approximate $Z^{2}$ dependence and is therefore suited to directly reveal atomic sites of single metal atoms on graphene. Details of the method and first results have been reported previously. ${ }^{[32]}$ Herein, we present an indepth study of the behavior of Au atoms evaporated onto pristine and hydrogenated graphene surfaces.

STEM image simulations of graphene layers were performed by using the Kirkland TEMSIM program. ${ }^{[33]}$ The multislice approximation was employed to calculate the electron wave function exiting the specimen. The simulations were carried out with the microscope parameters used to acquire the experimental STEM images. These parameters were: beam energy $60 \mathrm{keV}, \mathrm{Cs}-0.03 \mathrm{~mm}$, convergence angle $27 \mathrm{mrad}$, BF detector angular range 0-6 mrad, and HAADF angular range $70-210 \mathrm{mrad}$.

\section{Acknowledgements}

This work was supported by EPSRC (UK).

[1] K. S. Novoselov, A. K. Geim, S. V. Morozov, D. Jiang, Y. Zhang, S. V. Dubonos, I. V. Grigorieva, A. A. Firsov, Science 2004, 306, 666.

[2] S. Huh, J. Park, K. S. Kim, B. H. Hong, S. B. Kim, ACS Nano 2011 , 5, 3639.

[3] Y. Shi, K. K. Kim, A. Reina, M. Hofmann, L.-J. Li, J. Kong, ACS Nano 2010, 4, 2689.
[4] R. Erni, M. D. Rossell, M.-T. Nguyen, S. Blankenburg, D. Passerone, P. Hartel, N. Alem, K. Erickson, W. Gannett, A. Zettl, Phys. Rev. B 2010, 82, 165443.

[5] D. C. Elias, R. R. Nair, T. M. G. Mohiuddin, S. V. Morozov, P. Blake, M. P. Halsall, A. C. Ferrari, D. W. Boukhvalov, M. I. Katsnelson, A. K. Geim, K. S. Novoselov, Science 2009, 323, 610.

[6] K. T. Chan, J. B. Neaton, M. L. Cohen, Phys. Rev. B 2008, 77, 235430.

[7] Y. Mao, J. Yuan, J. Zhong, J. Phys.: Condens. Matter 2008, 20, 115209.

[8] H. Sevincli, M. Topsakal, E. Durgun, S. Ciraci, Phys. Rev. B 2008, 77, 195434.

[9] I. Suarez-Martinez, A. Felten, J. J. Pireaux, C. Bittencourt, C. P. Ewels, J. Nanosci. Nanotechnol. 2009, 9, 6171.

[10] B. Uchoa, C. Y. Lin, A. H. Castro Neto, Phys. Rev. B 2008, 77, 035420

[11] O. U. Akturk, M. Tomak, Phys. Rev. B 2009, 80, 085417.

[12] R. Varns, P. Strange, J. Phys.: Condens. Matter 2008, 20, 225005.

[13] O. Leenaerts, B. Partoens, F. M. Peeters, Microelectron. J. 2009, $40,860$.

[14] Y. Gan, L. Sun, F. Banhart, Small 2008, 4, 587.

[15] H. Pinto, R. Jones, J. P. Goss, P. R. Briddon, Phys. Status Solidi A 2010, 207, 2131.

[16] K. M. McCreary, K. Pi, A. G. Swartz, W. Han, W. Bao, C. N. Lau, F. Guinea, M. I. Katsnelson, R. K. Kawakami, Phys. Rev. B 2010, $81,115453$.

[17] J. Lee, K. S. Novoselov, H. S. Shin, ACS Nano 2011, 5, 608.

[18] D. W. Boukhvalov, M. I. Katsnelson, Appl. Phys. Lett. 2009, 95, 023109.

[19] J. C. Charlier, L. Arnaud, I. V. Avilov, M. Delgado, F. Demoisson, E. H. Espinosa, C. P. Ewels, A. Felten, J. Guillot, R. Ionescu, R. Leghrib, E. Llobet, A. Mansour, J. J. Pireaux, F. Reniers, I. Suarez-Martinez, G. E. Watson, Z. Zanolli, Nanotechnology 2009, 20, 375501.

[20] A. V. Krasheninnikov, P. O. Lehtinen, A. S. Foster, P. Pyykkö, R. M. Nieminen, Phys. Rev. Lett. 2009, 102, 126807.

[21] Z. Zhou, F. Gao, D. W. Goodman, Surf. Sci. 2010, 604, L31.

[22] Y. Okamoto, Chem. Phys. Lett. 2006, 420, 382.

[23] R. Muszynski, B. Seger, P. V. Kamat, J. Phys. Chem. C 2008, 112, 5263.

[24] W. Hong, H. Bai, Y. Xu, Z. Yao, Z. Gu, G. Shi, J. Phys. Chem. C 2010, $114,1822$.

[25] Z. Lee, K.-J. Jeon, A. Dato, R. Erni, T. J. Richardson, M. Frenklach, V. Radmilovic, Nano Lett. 2009, 9, 3365.

[26] G. Goncalves, P. A. A. P. Marques, C. M. Granadeiro, H. I. S. Nogueira, M. K. Singh, J. Gracio, Chem. Mater. 2009, 21, 4796.

[27] P. V. Kamat, J. Phys. Chem. Lett. 2009, 1, 520.

[28] K. Jasuja, J. Linn, S. Melton, V. Berry, J. Phys. Chem. Lett. 2010, 1, 1853.

[29] R. R. Nair, W. Ren, R. Jalil, I. Riaz, V. G. Kravets, L. Britnell, P. Blake, F. Schedin, A. S. Mayorov, S. Yuan, M. I. Katsnelson, H.-M. Cheng, W. Strupinski, L. G. Bulusheva, A. V. Okotrub, I. V. Grigorieva, A. N. Grigorenko, K. S. Novoselov, A. K. Geim, Small 2010, 6, 2877.

[30] J. O. Sofo, A. S. Chaudhari, G. D. Barber, Phys. Rev. B 2007, 75, 153401.

[31] X. Li, W. Cai, J. An, S. Kim, J. Nah, D. Yang, R. Piner, A. Velamakanni, I. Jung, E. Tutuc, S. K. Banerjee, L. Colombo, R. S. Ruoff, Science 2009, 324, 1312.

[32] R. Zan, U. Bangert, Q. Ramasse, K. S. Novoselov, Nano Lett. 2011, $11,1087$.

[33] E. J. Kirkland, Advanced Computing in Electron Microscopy, 1st ed., Plenum Press, New York 1998.

Received: June 13, 2011

Published online: August 24, 2011 